\title{
AN IMPROVEMENT ON THE UPPER BOUND OF THE NILPOTENCY CLASS OF SEMIDIRECT PRODUCTS OF $p$-GROUPS
}

\author{
LARRY J. MORLEY AND JOHN D. P. MELDRUM
}

\begin{abstract}
The semidirect product of a group $A$ by a group $B$ is necessarily nilpotent only in the case $A$ and $B$ are $p$-groups for the same prime $p, A$ is nilpotent of bounded exponent, and $B$ is finite. In an earlier paper Morley has established an upper bound on the class of a nilpotent semidirect product of an abelian $p$-group of bounded exponent by an arbitrary finite $p$-group. In this paper this result is improved by considering a direct product decomposition for $B$ and also by extending the results to give a new upper bound on the class in the most general case. The standard wreath product of $A$ by $B$ is a nilpotent semidirect product of relatively large class in the case $A$ and $B$ satisfy the conditions above, and this new bound improves the known results on the class of these wreath products.
\end{abstract}

1. Introduction. A group which is a semidirect product of $A$ by $B$ can be assumed nilpotent only with the conditions that $A$ and $B$ are $p$-groups for the same prime number $p, A$ is nilpotent of bounded exponent, and $B$ is finite (Baumslag [1]). The standard wreath product of $A$ by $B$ contains all semidirect products of $A$ by $B$ and the exact class of $A$ wr $B$ has been given by Liebeck [3] in the case $A$ and $B$ are abelian $p$-groups, $A$ is of bounded exponent and $B$ is finite. Meldrum [4] has given the class of $A$ wr $B$ in the case $A$ is nilpotent of exponent $p$ and $B$ is finite abelian. Morley [5] derives an upper bound for the class of a group which is a semidirect product of an abelian p-group of exponent $p^{n+1}$ by an arbitrary finite $p$-group. In this paper, an improvement in the upper bound of [5] is established and the new bound is also extended to the most general case of a nilpotent semidirect product of p-groups. The improvement in the bound is accomplished by considering a direct product factorization of the group $B$.

2. Notation and preliminary results. The notation and definitions used in this paper agree with, but in cases generalize, those in [5]. $\left(g_{1}, \ldots, g_{n}\right)$ and $\left(g_{1},(n-1) g_{2}\right)$ indicate commutator elements of length $n$, the second one having the last $n-1$ entries all $g_{2}$. An arbitrary ascending central series (see [2]) of the group $G$ is denoted $G_{0}<G_{1}<\cdots$ and the well-known lower

Received by the editors August 12, 1975 and, in revised form, February 27, 1976.

AMS (MOS) subject classifications (1970). Primary 20D15, 20E15, 20F25; Secondary $20 \mathrm{~F} 35$.

Key words and phrases. Nilpotency class, lower central series, semidirect products, direct product decomposition, $p$-groups, wreath products.

Copyright $\diamond 1977$. American Mathematical Society 
central series is written $G_{(i)}, i=0,1,2, \ldots$ The nilpotency class of $G$ is denoted $\mathrm{Cl}(G)$ and $\mathrm{Cl}(G)=L$ iff $G_{(L+1)}=E$ and $G_{(L)} \neq E, E$ the trivial subgroup containing the identity element only. If $H_{i}, i=1, \ldots, n$, are subgroups of $G$, then $\left(H_{1}, \ldots, H_{n}\right)$ denotes the subgroup of $G$ generated by $\left\{\left(h_{1}, \ldots, h_{n}\right) \mid h_{i} \in H_{i}\right\}$. The well-known commutator identities

$$
(x, y z)=(x, z)(x, y)(x, y, z), \quad(x y, z)=(x, z)(x, z, y)(y, z),
$$

are used in the proof of Theorem 3.1.

For an extension $W$ of $A$ by $B$ which is a semidirect product we assume both $A$ and $B$ are subgroups of $W$.

If $B=B_{1} \times B_{2} \times \cdots \times B_{m}$ is a direct product of finite $p$-groups, then $B_{k, 0}<\cdots<B_{k, L(k)}$ denotes an ascending central series of $B_{k}$ contained in $B$. Since $B_{k, i} / B_{k, i-1}$ is a finite abelian $p$-group, $B_{k, i}$ contains a minimal independent set of generators modulo $B_{k, i-1}$. The elements of such a generating set are written $\left\{b_{k, i, j}\right\}, j=1, \ldots, r(k, i), r(k, i)$ being the cardinality of the generating set which is called the rank of the factor group.

Definition 2.2. Let $p(k, i, j), 1 \leqslant j \leqslant r(k, i)$, denote the descending prime power orders of the cyclic groups in the decomposition of $B_{k, i} / B_{k, i-1}, k=1$, $\ldots, m$ and $i=1, \ldots, L(k)$, for a specified ascending central series of length $L(k)$ for $B_{k}$. Define $L=\max _{1 \leqslant k \leqslant m} L(k)$ and for $L(k)<i \leqslant L$ set $p(k, i, j)$ $=1$ and $r(k, i)=0$. Then we define

$$
\begin{gathered}
\lambda_{k i}=\sum_{j=1}^{r(k, i)}(p(k, i, j)-1), \quad 1 \leqslant k \leqslant m \text { and } 1 \leqslant i \leqslant L, \\
d(k, t, s)=\prod_{j=t+1}^{s} p(k, j, 1), \quad 1 \leqslant k \leqslant m, 1 \leqslant s \leqslant L, \text { and } 0 \leqslant t \leqslant s,
\end{gathered}
$$

and

$$
P_{k}\left(y_{1}, \ldots, y_{s}\right)=\sum_{t=1}^{s} d(k, t, s) y_{t} \text { for } s=1,2, \ldots, L
$$

The multivariable linear polynomials $P_{k}\left(y_{1}, \ldots, y_{s}\right)$ have coefficients determined by the exponents of the factor groups $B_{k, i} / B_{k, i-1}$ and the $\lambda_{k i}$ are dependent upon the complete cycle structure of these factor groups.

3. The upper bound. In the theorems of this section $B=B_{1} \times \cdots \times B_{m}$ is a direct product of finite $p$-groups. The terms expressed are defined using arbitrary but specified ascending central series for the respective direct factors of $B$ using Definition 2.2.

TheOREM 3.1. Let $W$ be a semidirect product of $A$ by $B, A$ abelian of exponent $p^{n+1}$. Then

$$
\mathrm{Cl}(W) \leqslant \sum_{k=1}^{m} P_{k}\left(\lambda_{k 1}, \ldots, \lambda_{k L(k)}\right)+n(p-1) p^{-1} \max _{1 \leqslant k \leqslant m} d(k, 0, L(k))+1 .
$$


Proof. Let

$$
c=\sum_{k=1}^{m} P_{k}\left(\lambda_{k 1}, \ldots, \lambda_{k L(k)}\right)+n(p-1) p^{-1} \max _{1 \leqslant k \leqslant m} d(k, 0, L(k))+1 .
$$

We will use induction on $m$. The result for $m=1$ is given in [5, Theorem 4.10]. So assume that the result holds for $m-1$.

Assume $\mathrm{Cl}(W)>c$ and obtain a contradiction. Without loss of generality we assume $\mathrm{Cl}(W)=c+1$ and choose $e \neq w \in W_{(c+1)}$. By [5, Corollary 3.4], $c>L=\mathrm{Cl}(B)$ and $w=\left(x, b_{0}, b_{1}, \ldots, b_{c-q}\right)$ where $b_{0} \in B_{(q)}$ and $b_{i}$ $\in B, 1 \leqslant i \leqslant c-q$ and $1 \leqslant q \leqslant L$. By the basic commutator identities, using the fact that $A$ is abelian and $W_{(c+2)}=E$, the map $b_{i} \rightarrow\left(x, b_{0}, b_{1}, \ldots\right.$, $\left.b_{c-q}\right)$ is a homomorphism for each $i, 0 \leqslant i \leqslant c-q$. So we may assume that $b_{i} \in B_{k}$ for some $k=k(i), 0 \leqslant i \leqslant c-q$. Since $\left(B_{k}, B_{l}\right)=E$ for $k \neq l$, [5, Lemma 4.6] allows us to assume that all the elements $b_{i}$ from a given $B_{k}$ follow each other. Let the number of entries from $B_{k}$ be $c_{k}$. Then $c=\sum_{k=1}^{m} c_{k}$.

Without loss of generality we may assume that

$$
c_{m}>P_{m}\left(\lambda_{m 1}, \ldots, \lambda_{m L(m)}\right)+\left(n-t_{m}\right)(p-1) p^{-1} d(m, 0, L(m)),
$$

where $t_{m}$ is minimal subject to this inequality holding. By [5, Theorem 4.10] applied to $A \cdot B_{m}$, we may assume that $\left(x, b_{1}, \ldots, b_{c_{m}}\right)$ has order dividing $p^{t_{m}}$, where $b_{i} \in B_{m}, 1 \leqslant i \leqslant m$.

If $t_{m}=0$, then $w=e$ and the contradiction is obtained. So assume that $t_{m} \neq 0$. Then, by the minimality of $t_{m}$,

$$
\begin{aligned}
c^{\prime}=c-c_{m} \geqslant & \sum_{k=1}^{m-1} P_{k}\left(\lambda_{k 1}, \ldots, \lambda_{k L(k)}\right) \\
& +\left(t_{m}-1\right)(p-1) p^{-1} \max _{1 \leqslant k \leqslant m-1} d(k, 0, L(k))+1 .
\end{aligned}
$$

Let $w^{\prime}=\left(x^{\prime}, b_{c_{m}+1}, \ldots, b_{c}\right)$ where $x^{\prime}=\left(x, b_{1}, \ldots, b_{c_{m}}\right)$. By the induction hypothesis on $m, w^{\prime}=e$ since $w^{\prime} \in A \cdot\left(B_{1} \times \cdots \times B_{m-1}\right)$. This gives the final contradiction.

The proof of the following theorem is an adaption of the proof of Theorem 5.12 of [4].

Theorem 3.2. Let $W$ be a semidirect product of $A$ by $B=B_{1} \times \cdots \times B_{m}, A$ a nilpotent p-group of class $c$ and $B_{k}$ a finite p-group for each $k=1, \ldots, m$. Suppose $A_{0}<A_{1}<\cdots<A_{c}$ is an ascending central series of $A$. If $A_{j} / A_{j-1}$ has exponent $p^{n(j)}$ for $1 \leqslant j \leqslant c$, then

$$
\begin{aligned}
\mathrm{Cl}(W) \leqslant & c\left(\sum_{k=1}^{m} P_{k}\left(\lambda_{k 1}, \ldots, \lambda_{k L(k)}\right)\right) \\
& +\left(\sum_{j=1}^{c}(n(j)-1)\right)(p-1) p^{-1} \max _{1 \leqslant k \leqslant m} d(k, 0, L(k))+c .
\end{aligned}
$$


Proof. We proceed by induction on $c$. Theorem 3.1 is just the statement of this result for $c=1$ so we let $c>1$. Define

$$
\begin{aligned}
t(j)= & \sum_{k=1}^{m} P_{k}\left(\lambda_{k 1}, \ldots, \lambda_{k L(k)}\right) \\
& +(n(j)-1)(p-1) p^{-1} \max _{1 \leqslant k \leqslant m} d(k, 0, L(k))+1,
\end{aligned}
$$

Now $A_{1}$ is a normal subgroup of $W$ and $W / A_{1}$ is a semidirect product of $A / A_{1}$ by $B$. By the induction hypothesis $\mathrm{Cl}\left(W / A_{1}\right) \leqslant \sum_{j=2}^{c} t(j)$ since $\left(A_{j} / A_{1}\right) /\left(A_{j-1} / A_{1}\right)$ is isomorphic to $A_{j} / A_{j-1}$ for $2 \leqslant j \leqslant c$. Thus we have that $W_{(t)} \subseteq A_{1}$ for $t=\sum_{j=2}^{c} t(j)+1$. The result now follows from Theorem 3.1 since $A_{1}$ is contained in the centre of $A$ implies $\left(A_{1}, k W\right) \subseteq\left(A_{1}, k B\right)$.

\section{REFERENCES}

1. G. Baumslag, Wreath products and p-groups, Proc. Cambridge Philos. Soc. 55 (1959), 224-231. MR 21 \#4179.

2. P. Hall, Nilpotent groups, Canadian Math. Congress, Summer Seminar, Univ. of Alberta, 1957.

3. H. Liebeck, Concerning nilpotent wreath products, Proc. Cambridge Philos. Soc. 58 (1962), 443-451. MR 25 \#3087.

4. J. D. P. Meldrum, On nilpotent wreath products, Proc. Cambridge Philos. Soc. 68 (1970), 1-15. MR 41 \#5500.

5. L. Morley, Bounds on the nilpotency class of certain semidirect products, Trans. Amer. Math. Soc. 159 (1971), 381-390. MR 44 \# 1737.

Department of Mathematics, Western Illinois University, Macomb, Illinois 61455 (Current address of L. J. Morley)

Department of Mathematics, University of Edinburgh, Edinburgh EH8 9YL, Great BRITAIN

Current address: (J. D. P. Meldrum): Mathematics Department, James Clark Maxwell Building, The King's Buildings, Mayfield Road, Edinburgh EH9 3JZ, Scotland 